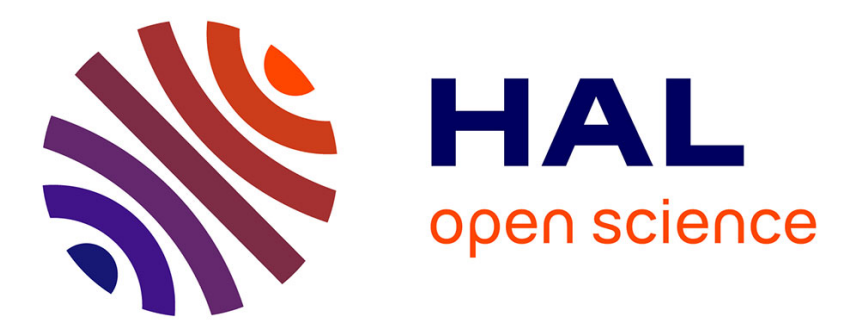

\title{
Sur un préamplificateur laser femtoseconde de conception optique simple
}

\author{
C. Hirlimann
}

\section{To cite this version:}

C. Hirlimann. Sur un préamplificateur laser femtoseconde de conception optique simple. Revue de Physique Appliquée, 1987, 22 (12), pp.1673-1676. 10.1051/rphysap:0198700220120167300 . jpa00245727

\section{HAL Id: jpa-00245727 https://hal.science/jpa-00245727}

Submitted on 1 Jan 1987

HAL is a multi-disciplinary open access archive for the deposit and dissemination of scientific research documents, whether they are published or not. The documents may come from teaching and research institutions in France or abroad, or from public or private research centers.
L'archive ouverte pluridisciplinaire HAL, est destinée au dépôt et à la diffusion de documents scientifiques de niveau recherche, publiés ou non, émanant des établissements d'enseignement et de recherche français ou étrangers, des laboratoires publics ou privés. 
Classification

Physics Abstracts

42.60B

\title{
Sur un préamplificateur laser femtoseconde de conception optique simple
}

\author{
C. Hirlimann
}

Université Pierre et Marie Curie, Laboratoire de Physique des Solides, Spectroscopie des Solides (U.A. 154), 4, place Jussieu, Tour 13-2, 75252 Paris Cedex 05, France

(Reçu le 9 juin 1987, révisé le 16 juillet 1987, accepté le 24 août 1987)

\begin{abstract}
Résumé. - Nous avons réalisé un dispositif optique simple et compact permettant d'amplifier des impulsions lumineuses d'une durée de $100 \mathrm{fs}$ en minimisant l'importance de la dispersion de la vitesse de groupe. Le montage permet le passage multiple des impulsions dans une même région de gain réalisée dans un jet et un gain total de l'ordre de 10000 pour six passages.
\end{abstract}

Abstract. - We have designed a very simple, compact, all reflective optical set up for a multipass laser amplifier. This scheme allows an amplification factor of up to 10000 for $100 \mathrm{fs}$ light pulses, while minimizing group velocity dispersion.

\section{Introduction.}

Dès les débuts du développement des sources laser délivrant des impulsions lumineuses en régime femtoseconde, la nécessité s'est faite sentir de pouvoir disposer d'amplificateurs permettant d'élever la puissance disponible bien au-delà des quelques kilowatts alors accessibles [1]. D'une part pour pouvoir accéder à l'étude des processus optiques non linéaires, mais aussi parce que, les oscillateurs existants n'étant pas accordables, il est apparu très tôt que la façon la plus simple de faire de la spectroscopie était d'engendrer des impulsions de lumière blanche [2]. Ces impulsions à très large bande spectrale ne peuvent être obtenues que pour des puissances crête de l'ordre du gigawatt.

Les amplificateurs les plus utilisés ont d'abord été constitués d'une série de cuves de colorant pompées transversalement ou longitudinalement [2] ; puis est apparue la possibilité d'utiliser des jets de colorant permettant à la fois de minimiser la distorsion temporelle des impulsions, par dispersion de la vitesse de groupe et d'assurer une meilleure qualité modale au faisceau amplifié [3-4]. Nous avons développé un montage optique simple assurant l'amplification d'impulsions, d'une durée d'une centaine de femtosecondes, par multipassage de la lumière dans un jet de colorant.

\section{Schéma optique et techniques expérimentales.}

Ainsi qu'il est indiqué sur la figure 1 , le système optique employé est constitué de trois miroirs. Deux

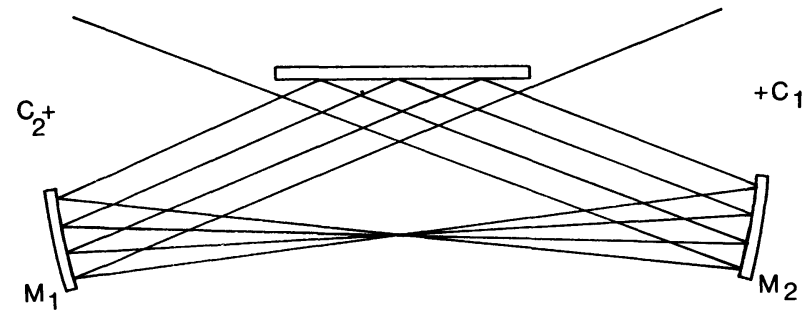

Fig. 1. - Schéma optique du préamplificateur à jet. $\mathrm{C}_{1}$ et $\mathrm{C}_{2}$ représentent les centres de courbure des miroirs concaves $M_{1}$ et $M_{2}$.

[Schematic of the optical set up for the jet laser preamplifier. $C_{1}$ and $C_{2}$ stand for the centres of the pair of concave mirrors.]

miroirs concaves identiques $\mathrm{M}_{1}$ et $\mathrm{M}_{2}$ ayant leur centre de courbure placé respectivement en $\mathrm{C}_{1}$ et $\mathrm{C}_{2}$ assurent la focalisation et la collimation du faisceau. Un faisceau tombant sur le miroir $M_{1}$ est d'abord focalisé puis recollimaté par le miroir $\mathrm{M}_{2}$; il se réfléchit alors sur un miroir plan placé orthogonalement à l'axe de symétrie de la figure. Cette dernière réflexion fait subir au faisceau une translation latérale qui permet à la lumière d'être focalisée plusieurs fois en un même point. La position des centres $C_{1}$ et $C_{2}$ n'est pas critique, elle doit simplement être choisie de sorte à libérer suffisamment d'espace libre autour du point de concours pour pouvoir y placer le jet de colorant. L'intersection des faisceaux au point de focale, que l'on peut visualiser avec une feuille transparente, ne 
peut être obtenue que par un ajustement soigneux de la distance entre $M_{1}$ et $\mathbf{M}_{2}$ qui dépend de la focale et de la déviation hors d'axe. Le nombre de passages de la lumière dans le système peut aisément être changée par une simple translation du miroir plan [5].

Nous avons employé dans notre système des miroirs à revêtement d'argent protégé ayant une réflectivité de $98 \%$. Les miroirs sphériques ont une longueur focale de $150 \mathrm{~mm}$ et le miroir plan a une longueur de $30 \mathrm{~mm}$. L'alignement est tel que les faisceaux sont étalés dans une nappe de $6^{\circ}$. Le faisceau du laser d'excitation fait dans le plan horizontal un angle de $10^{\circ}$ avec le faisceau médian de la nappe.

Les impulsions lumineuses à amplifier sont produites par un laser à colorant à blocage de modes par collision d'impulsion (C.P.M.) [6]. Elles ont une durée d'environ $100 \mathrm{fs}$, une énergie de $50 \mathrm{pJ}$ et une période de récurrence de $10 \mathrm{~ns}$. La région de gain, sur laquelle nous reviendrons, est excitée par le faisceau d'un laser Nd: YAG doublé en fréquence (longueur d'onde d'émission $=532 \mathrm{~nm}$ ) dont la fréquence de répétition est de $10 \mathrm{~Hz}$. La synchronisation des deux lasers a été réalisée de la manière suivante : les impulsions d'horloge du YAG arment dix fois par seconde une bascule. Une photodiode placée sur une réflexion du C.P.M. déclenche la bascule par la première impulsion lumineuse qu'elle reçoit après l'armement. L'impulsion électrique engendrée par la bascule déclenche à son tour la cellule de Pockels du laser après être passée dans une ligne à retard variable assurant la synchronisation. Une mesure simultanée des impulsions lumineuses du C.P.M. et du YAG à l'aide d'une photodiode rapide et d'un oscilloscope à échantillonnage a montré que la gigue est inférieure ou égale au temps de réponse de l'ensemble de mesure égal à 800 ps.

La région de gain est un jet d'environ un millimètre d'épaisseur produit par une buse d'acier inoxydable réalisée localement. L'éthylène glycol a été choisi comme solvant visqueux du colorant employé. Dix à vingt pour-cent de glycérine y ont été dissous pour augmenter encore la viscosité et assurer l'écoulement laminaire du jet et donc sa bonne qualité optique [7]. La sulforhodamine 101 a servi de milieu actif pour le gain car son émission est maximum à $608 \mathrm{~nm}$ lorqu'on l'excite à $532 \mathrm{~nm}$ ce qui est suffisamment proche de la longueur d'onde à amplifier de $620 \mathrm{~nm}$. Sa concentration a été ajustée pour absorber $80 \%$ de la lumière de pompe. Une lentille de $30 \mathrm{~cm}$ de longueur focale, concentre la lumière de pompe sur le jet sous la forme d'une tache de diamètre $1 \mathrm{~mm}$. Cette disposition assure un bon accord géométrique entre le volume de gain et les volumes focaux des différents faisceaux. L'emploi d'une lentille de plus courte focale détruit en partie l'accord géométrique et abaisse l'amplification obte- nue. Une lame demi-onde est placée sur le trajet de la lumière excitatrice pour rendre sa polarisation parallèle à celle de la lumière à amplifier.

Un absorbant saturable est situé à la sortie de la partie amplificatrice. Il est constitué d'un jet standard de malachite verte dissoute dans de l'éthylène glycol. Ce jet est placé au foyer d'une lunette de grandissement 1 utilisant deux lentilles de longueur focale $3 \mathrm{~cm}$. La transmittance à faible signal est fixée $1,5 \times 10^{-3}$. Le rôle de l'absorbant saturable est double: d'une part il absorbe les impulsions de l'oscillateur qui ne sont pas amplifiées et d'autre part il atténue l'émission spontanée amplifiée. La malachite verte est choisie pour son temps de recouvrement court, de 2 ps [8], qui minimise la part d'émission spontanée qui n'est pas absorbée après le passage de l'impulsion femtoseconde.

Un microwattmètre à photodiode (Coherent Radiation CR212) a été utilisé pour mesurer la puissance moyenne des diverses quantités nécessaires à la détermination du gain : lumière totale, émission spontanée, impulsions non amplifiées résiduelles. Nous avons pris la précaution d'atténuer fortement les intensités mesurées pour assurer l'utilisation de la photodiode dans sa zone de réponse linéaire.

La mesure de la durée des impulsions lumineuses a été faite par la technique d'auto-corrélation par génération d'harmonique de second ordre, non colinéaire dans un cristal de K.D.P. convenablement orienté, de $200 \mu \mathrm{m}$ d'épaisseur [9].

\section{Résultats.}

Le tableau I résume les valeurs de gain obtenues pour des nombres de passages différents. L'énergie disponible par impulsion est de l'ordre de $0,3 \mu \mathrm{J}$ dans une configuration à 6 passages. L'absorbant saturable n'introduit pas d'atténuation supplémentaire mesurable. Les valeurs de gain obtenues ne sont pas à prendre en valeur absolue, de plus fortes valeurs ayant été atteintes par un meilleur réglage du système. Il convient cependant de remarquer que le gain moyen par passage décroît lorsque l'on passe de 4 à 6 passages. Deux raisons peuvent être invoquées pour expliquer ce fait. La première est liée à l'augmentation de l'émission spontanée amplifiée qui croît avec le nombre de passages et qui entre de plus en plus fortement en compétition avec l'amplification. La deuxième raison est géométrique : le

Tableau I.

Nombre de passages

\begin{tabular}{lrrrr}
\hline & 4 & 5 & 6 \\
Gain total & 1300 & 2 & 300 & 4 \\
Gain moyen par passage & 6 & 4,7 & 4 \\
\hline
\end{tabular}


temps de parcours de la lumière entre deux passages dans la région de gain est de l'ordre de $2 \mathrm{~ns}$; à six passages le temps de vol est donc de $10 \mathrm{~ns}$, de sorte que l'impulsion amplifiée voit le début et la fin du pompage donc des gains moindres.

Ceci se traduit, à la sortie de l'absorbant saturable, par une variation du rapport entre l'émission spontanée amplifiée et l'impulsion amplifiée qui varie de $1 \%$ à 4 passages à $12 \%$ à 6 passages.

Les impulsions de l'oscillateur ayant une récurrence de $10 \mathrm{~ns}$, il n'est pas possible d'augmenter le nombre de passages dans le système, si l'on veut éviter d'amplifier l'impulsion suivante.

Ces mesures ont été faites en assurant, à l'aide de la lame demi-onde sur le faisceau de pompe, le parallélisme des polarisations de l'impulsion amplifiée et de l'impulsion d'excitation. Aucune donnée expérimentale sur le temps de réorientation de la sulforhodamine en solution dans le glygol n'a pu être trouvée dans la littérature. Si l'on suppose le temps de réorientation désordonnée de la polarisation du milieu de gain courte devant le temps caractéristique d'amplification, le gain par unité d'épaisseur de colorant disponible, lorsque les polarisations de la lumière sont croisées, est réduit par un facteur 3. Pour un gain par passage de 4 en polarisations parallèles, le gain total à 6 passages n'est plus alors que de $\left(4^{1 / 3}\right)^{6}=16$ environ en polarisations croisées. Un tel gain est largement en dessous des possibilités de mesure de notre microwattmètre et explique que nous n'ayons pu mesurer aucune amplification en polarisation croisée. Cette remarque prend toute son importance si l'on envisage d'utiliser comme

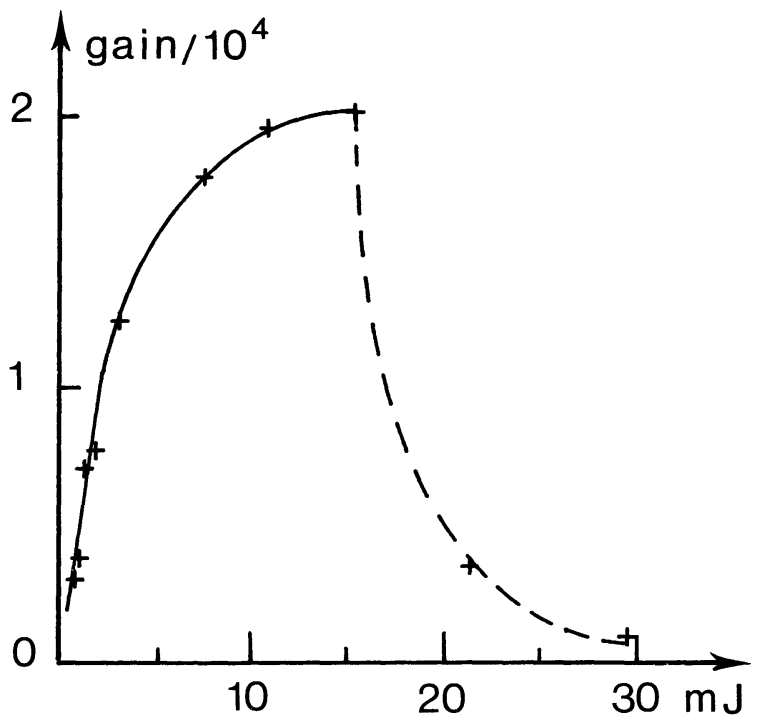

Fig. 2. - Variation du gain total obtenu pour six passages d'impulsions de $100 \mathrm{fs}$, en fonction de l'énergie des impulsions d'excitation du gain.

[Total gain for six passes of $100 \mathrm{fs}$ eight pulses, versus pumping energy.] source d'excitation un laser dont la lumière n'est pas polarisée et où l'on risque donc de chauffer inutilement le milieu de gain.

La figure 2 montre la variation du gain total, pour six passages, en fonction de l'énergie des impulsions d'excitation. Trois régimes peuvent être distingués. Jusqu'à $2 \mathrm{~mJ}$ le comportement du gain est linéaire, puis on entre dans un régime de saturation qui se poursuit jusqu'à une énergie de $15 \mathrm{~mJ}$. Cette saturation reflète la compétition entre le processus d'amplification de l'impulsion du C.P.M. et l'amplification de l'émission spontanée. Au-delà de $15 \mathrm{~mJ}$ le gain s'écroule. Cet effet est vraisemblablement dû à un début d'effet laser entre les faces du jet de colorant. Cette courbe montre qu'un gain total de 10000 peut être atteint en régime linéaire, résultat qui est équivalent à ce qui a été observé pour le montage de la référence [4].

Nous avons reporté sur la figure 3 les traces d'autocorrélation pour les impulsions avant et après amplification. On observe un doublement de la

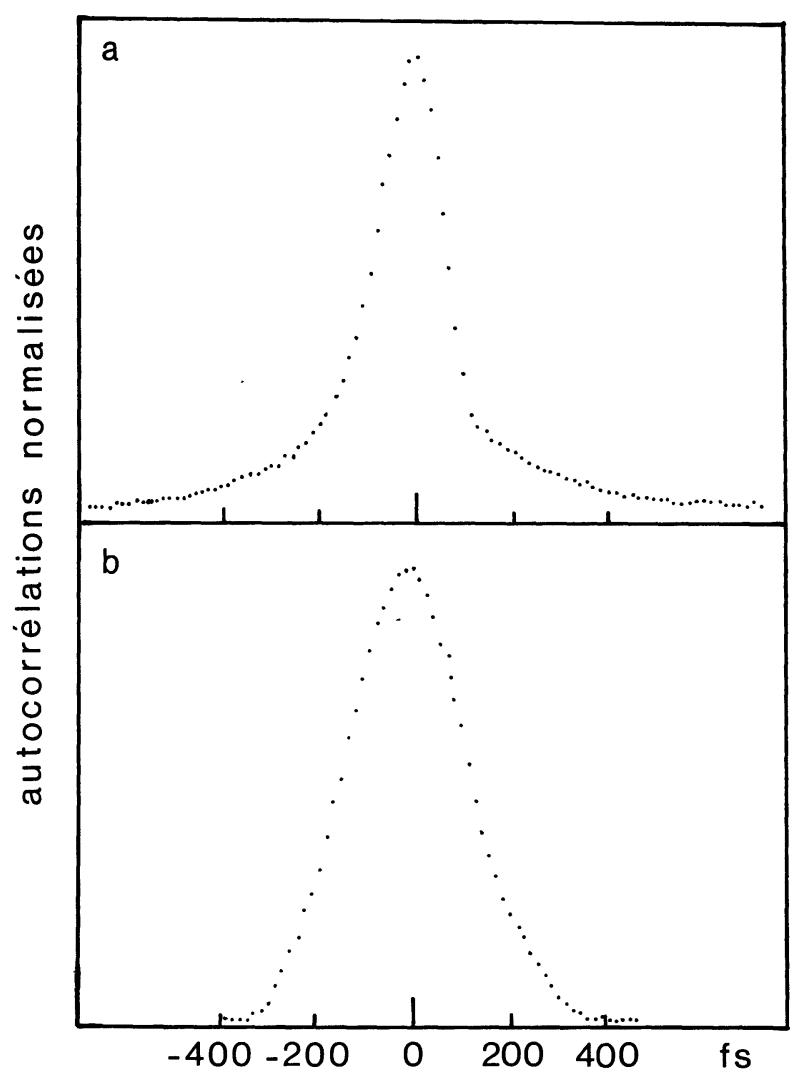

Fig. 3. - Traces d'auto-corrélation des impulsions lumineuses. a) à la sortie de l'oscillateur : la durée est de 100 fs en assumant une enveloppe de forme sécante hyperbolique carrée. b) à la sortie de l'amplificateur : la durée est de $200 \mathrm{fs}$ en faisant la même hypothèse.

[Autocorrelation traces for the light pulses. a) output of the C.P.M. : the duration is $100 \mathrm{fs}$ assuming squared hyperbolic secante shape. b) output of the amplifier : the duration is $200 \mathrm{fs}$, under the same assumption.] 
durée des impulsions par passage à travers le système. Ceci est compatible avec l'hypothèse d'un élargissement temporel dû à la dispersion de la vitesse de groupe à la traversée des jets et des lentilles.

Le système que nous avons présenté est extrêmement simple sur le plan optique, il présente néanmoins deux défauts. Le premier est lié à l'utilisation hors d'axe de miroirs sphériques dont l'astigmatisme dégrade la qualité optique du faisceau. L'emploi de miroirs paraboliques hors d'axe devrait permettre de remédier à cet inconvénient. Par construction, ce montage n'introduit que peu de pertes sur l'émission spontanée amplifiée qui entre donc facilement en compétition avec le processus d'amplification. Pour surmonter cette difficulté, nous proposons la modification suivante : le miroir plan du système constitue un plan de symétrie, on peut donc le remplacer par une paire de miroirs concaves en position de symétrie par rapport aux premiers ce qui permet de disposer d'un deuxième point de focale commun. Ce point commun peut être utilisé pour découpler l'impulsion amplifiée de la superadiance en introduisant un jet d'absorbant saturable ou un filtrage spatial.

Nous nous proposons, dans un avenir proche, de faire suivre le préamplificateur d'un compresseur temporel à prisme pour compenser l'élargissement temporel des impulsions par dispersion de vitesse de groupe. Cette solution présente l'avantage de permettre de séparer la superadiance résiduelle des impulsions amplifiées et donc d'améliorer le contraste.

\section{Remerciements.}

L'auteur tient à remercier MM. J. F. Morhange et O. Seddiki pour leur cruciale contribution à ce travail, M. G. Mouget qui a réalisé l'électronique de synchronisation entre les lasers ainsi que $\mathbf{M}$. B. Barrau pour la réalisation des parties mécaniques du montage.

\section{Bibliographie}

[1] Fork, R. L., Shank, C. V. and Yen, R., Appl. Phys. Lett. 41 (1982) 223.

[2] Fork, R. L., Shank, C. V., Hirlimann, C., Yen, R. and Tombinson, V. J., Opt. Lett. 8 (1983) 1.

[3] Gustafson, T. L. and Roberts, D. M., Opt. Comm. 43 (1982) 141.

[4] Knox, W. H., Downer, M. C., Fork, R. L. and Shank, C. W., Opt. Lett. 9 (1984) 552.

[5] Hirlimann, C., Seddiki, O., Morhange, J. F., Mounet, R. and Goddi, A., Opt. Comm. 59 (1986) 52.
[6] Fork, R. L., Green, B. I. and Shank, C. V., Appl. Phys. Lett. 38 (1981) 671.

[7] Fork, R. L. and BEISSER, F. A., communication privée.

[8] Ippen, E. P., Shank, C. V., Bergman, A., Chem. Phys. Lett. 38 (1976) 611.

[9] Ippen, E. P., Shank, C. V., Appl. Phys. Lett. 27 (1975) 488. 